\title{
A New Mobility Model for Ad Hoc Networks in Disaster Recovery Areas
}

\author{
https://doi.org/10.3991/ijoe.v13i06.7106 \\ Saher Manaseer \\ The University of Jordan, Amman, Jordan \\ saher@ju.edu.jo \\ Afnan Alawneh \\ The University of Jordan, Amman, Jordan \\ a. alawneh@ ju.edu.jo
}

\begin{abstract}
Over the last decade, many researchers have focused on Mobile Ad Hoc Networks as the main communication method in disaster recovery situations. In these researches, there has been marginal focus on the mobility patterns of nodes in disaster recovery scenarios. In this paper, a deeper analysis has been performed on some of the main mobility models used in testing new protocols and a new mobility model is proposed to incorporate some neglected factors concerned with disaster recovery situations.
\end{abstract}

Keywords - MANETs, Disaster recovery, Mobility Model, Random Waypoint, Routing, Hexacell.

\section{$1 \quad$ Introduction}

Managing disasters and emergency situations requires accurate and precise messages delivery, on which sensitive decisions making in such scenarios depends [1]. Since most of the infrastructure of any communication is eradicated by the strike of the disaster, this can affect rescuing missions and recovery which are timely critical. The alternative should be planned and set for managing the post disaster situations and emergency issues.

Disaster perspective, is how people look at the disaster and what they believe that is most critical to be managed after the disaster, which affects the parameters that are chosen and taken into consideration in such situations, this can affect efficiency of disaster management which supposed to minimize losses in lives [2].

Communications play a key role in disasters recovery and emergency management situations. Ad hoc networks are infrastructure-less networks which make the main focus of researches is how to implement them in disasters recovery areas. In order for the study of disaster recovery situations to be more realistic and of higher credibility, the mobility model that describes node movement in the network area should be representative and reflects such scenarios. This is the motive behind the proposed model 
for Ad hoc networks that will be used as a model in decision-making in such situations [3] [4].

\section{$2 \quad$ Related works}

Disasters and emergency cases, such as hurricanes, earthquakes, attacks...etc., can be hardly predicted, moreover their severity can be hardly estimated [1]. Post management for emergency and disasters can be critical for the evacuation and rescuing, or any other process related to the recovery.

As an infrastructure-less network, MANETs are the best choice so far for such cases. Though their performance degrades in the case of end-to-end paths. The multipath routing protocols serve as a method to enhance the performance of the communication networks in such situations [5]. However, in disastrous situations, such protocols are considered to be complex due to the metrics of performance such as delay time, reliability of communication, the consumption of power and other metrics that make it difficult to build a system to solve management problems in such scenarios [6].

In [5] a MANETs multipath routing protocol was presented on the base of the biological attractor selection scheme, in which the author stated that this way could improve and enhance the flexibility of the network and make it adaptable to the dynamic changing environments. This has appositive effect on the packet-delivering ratio and on the overall performance and throughput.

COMVIVOR is communication framework for survivors in disaster or emergency situations, which considers the strategy of smart positioning to increase the speed of the information spreading process. This framework aims at positioning the special nodes in a better way to improve the propagation. The model shows that the smart positioning enhances the dynamics of propagation [1][7].

Most of the published work on disaster recovery using MANETs have tested networks using random mobility models. Random Waypoint is an example of these models. Besides being unrealistic, random mobility patterns do not accommodate the special conditions and limitations that are present in disaster areas. Hazards and lifethreatening factors do not participate when designing node mobility. Results extracted using random, uncontrolled and none calculated movement have less significance and are unable to represent enough indicators to help decision makers.

Moving into a threatened area of the network has significant impact on the availability of the nodes and hence effect the continuity of network functions. Moreover, even if the node is not shut down by external factors, the performance levels of nodes are highly threatened to be compromised by external factors such as heat and humidity, which are major factors that can jeopardize any electronic device.

Defining a special mobility model for such scenarios has major effects on many other aspects of networking. For instance, any new proposal for routing mechanisms that aim for disaster recovery cannot be evaluated properly under the commonly used mobility models such as Random waypoint model. The assumption of random locations of nodes is a critical issue that can significantly affect the efficiency of routing and rout cost calculation. Therefore, the proposed model intendeds to be more realis- 
tic and hence, produces more reliable routing decisions. In addition, the proposed mobility model increases the predictability of node locations in a way that can simplify route selection by knowing node location and cell business in advance.

Another possible area of enhancement by using the proposed model is media access control. By the definition of our model, contention levels are easily obtained by cell weight. As per the explanation of the basic functionality of the mobility model, the cell weight depends on the number of nodes in the cell alongside with other factors. Therefore, knowing the cell weight is usable as a decision making factor so that the media access control mechanism takes the cell business into account when deciding giving turns to nodes to use the channel. This is particularly true if nodes are classified according to their significance as senders in the network.

In another scenario, many studies have proposed frameworks and algorithms to transform the GPS maps into digital representations, in which to help in the emergency cases and disastrous situations, such algorithms suffer the consuming of time, where time is a very critical factor in evacuating or rescuing processes, plus the time and memory space required to save the data and analyze it [8].

Data collection and delivery is a critical issue in emergency cases, for this purpose; many studies have proposed frameworks and algorithm to minimize the delay of information transmission and enhance the data collection process. Opportunistic networks and Data tolerant networks were used for this purpose. In [9] the deployment of opportunistic protocols in disaster situation was analyzed. On the other hand an information system was proposed to handle sever failure in a disaster case with the existence of cloud computing [10]. DistreesNet is a wireless sensor network that has the ability to provide data collection in emergency cases [11].

Not only the delay of data delivery is caused in emergencies, but also the computing requirements to generate maps are relatively high. In [7], a system to generate a map for pedestrians in disaster situation was proposed. In which the collection of data and sharing messages without end-end networks, that doesn't rely on cloud computing..

\section{Proposed Model}

In any emergency, the need for communication increases significantly. Thus, this paper presents a model to help enhancing and repairing the destruction in the network and communication channels, using Ad Hoc Wireless Networks.

Main objective behind this model is to help the recovery and minimize time needed to reach the totally destroyed areas, since using an end-to-end will cause congestion and high load on the same paths available. Moreover this increases power consumption and increase delay time.

A disaster area is treated as an empty and open-space area. This assumption is behind the oversimplified scenarios when studying disaster recovery situations with MANETs. A deeper look at an earthquake area reveals that many factors should be taken into account. The aftershock of the earthquake should be analysed in a way that excludes the weak areas that are more likely to be hit again should are given smaller 
levels of acceptance as candidate residency option. On the other hand, regions that have higher accessibility from outside the area are the best choices and should have higher acceptance. It is important to remember that this analysis can be done in short time in some cases, but it might not be feasible in the case of disasters.

First, the proposed model will split the disaster area into grids. The area is going to be divided into a hexagonal grids, since transmission areas for the node is circular and the hexagonal is the closer shape to cover circular areas, and if the model is to be applied on mobile phones the hexagonal is the adopted shape for the cellular networks. After the area is divided, a path finding method should be taken into account to find the path that should be followed during node's movement in the disaster area, the nodes will be spread in the hexa-cells.

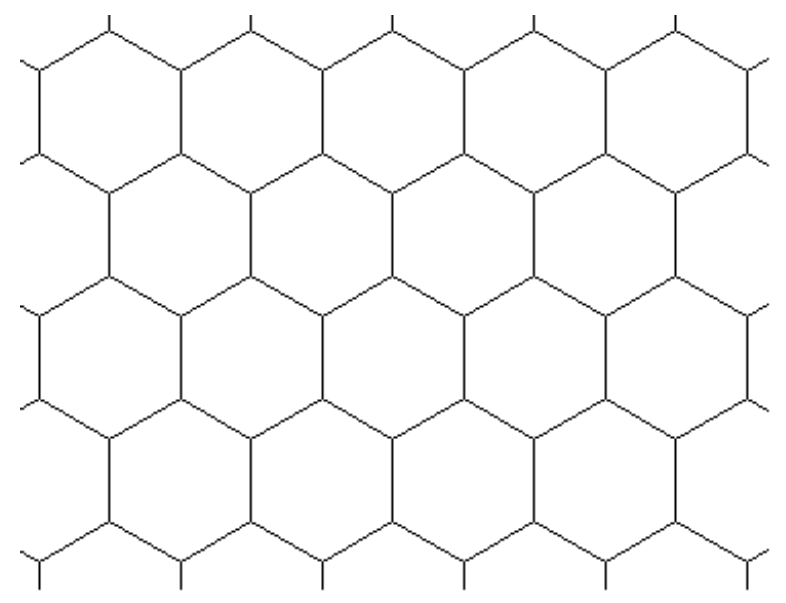

Fig. 1. Area into Hexa- Grids

Three common algorithms are known in finding the paths in such emergency situations: Breadth first search; this algorithm explores in all directions equally. The second algorithm is the Dijkstra's Algorithm that seeks the lower cost paths by avoiding the blocked ways due to the disaster, the trees, enemies near places and others obstacles. Lastly, The A* is a modified algorithm of the Dijkstra's. Where it was optimized for a single location.

Using such algorithms to filter the locked neighbouring cells that are impassable. Due to destruction, the weight of such cells will be the least, to decrease the possibility of entering those areas. A heuristic function can be applied to find the distance between two grids "areas".

Each grid cell has a weight that represents the priority of that cell. This weight will help the nodes to decide their paths and where to move in the destroyed area, the weight value will depend on the ability to use the grid cell, in other words if this place is safe to enter or not.

In order to decide the weight of any cell, the range of possible values depends on the factors that matter the most. For instance, the importance of the cell in reference to the area of the disaster is a major issue. For example, it might be beneficial to know 
the parts of the area that must be covered and, if possible, to cover them with a larger number of nodes to support higher rates of data transmission. In the case of this work, the weight depends on the desired number of nodes in any cell. For example, if a maximum of $\mathrm{N}$ nodes is desired, the range will be $[-\mathrm{N} \ldots \mathrm{N}]$. The weight will be set in both negative and positive values, the range proposed for this model falls between -10 to +10 . In this case, 10 nodes or less is the desired crowd.

Figure 2 shows the cell A is crowded, cell B is not crowded yet and can have more nodes, the grey represent the impassable grids.

The propagation of the nodes will start according the following:

A parameter that is defined as desired number of nodes (i.e. name of the defined parameter D). Figure 3 shows how the process works, where $\mathrm{W}=\mathrm{Weight}$ of the cell that the node is approaching or considering for routing and positioning purposes. $\mathrm{D}=$ the desired number of nodes.

At any given time, each node in the cell has a copy of the weight value. Having this value stored in each node has many advantages; one advantage is that this value will never be lost since all nodes have it. This is a very critical point since there is not central management in the network. The other advantage is that the value can be obtained by a simple communication with the nearest node inside the cell.

For the second stage, after the nodes take their places according to the cell weight in the grid, there should be a re-weighting algorithm that works in the case of new disaster shock or new emergency situation later after the first strike. In this case each new node enters the cell should send a broadcast message to the first hop neighbors. Upon the receipt of this broadcast, the weight value in each node should be reduced by 1 . After that, the value of weight is copied into the new node as well. In this case when the number of nodes increases in the cell the weight of the cell shall decrease in order to keep minimum nodes crowding in the cell.

To summarize the basic functionality of nodes in order to implement the proposed mobility model, Figure 4 shows the main steps.

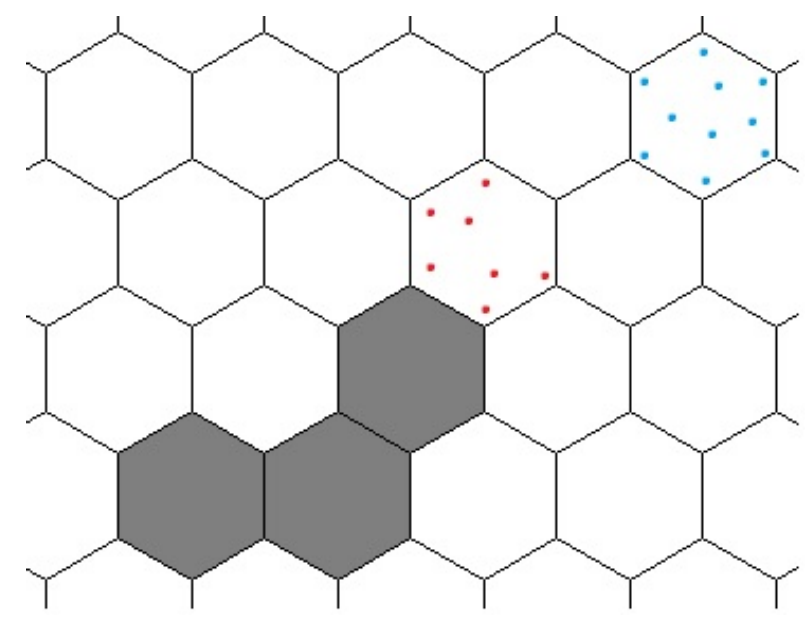

Fig. 2. Nodes in hexa Grid cells 


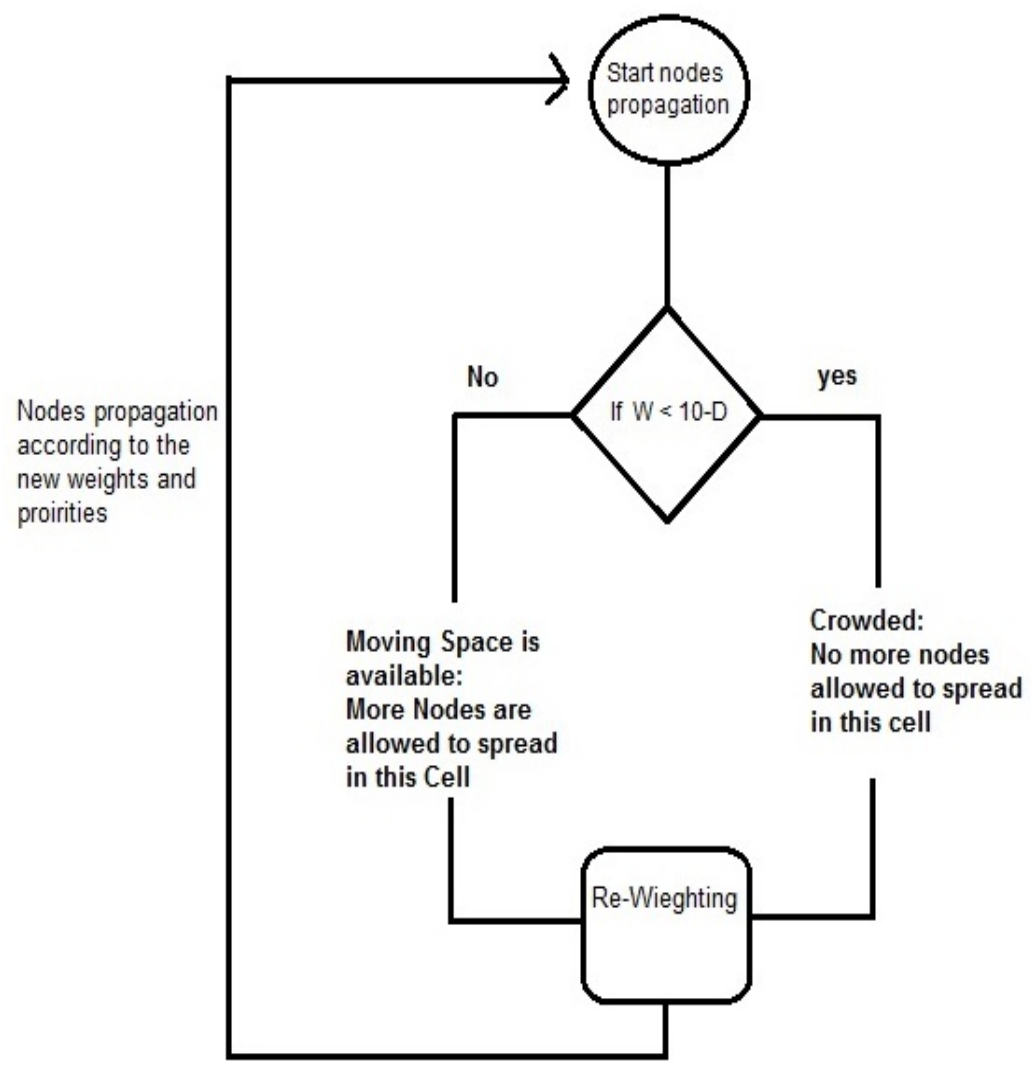

Fig. 3. Proposed Model

Step 0: for each of the neighboring cells, retrieve cell weight.

Step 1: chose the cell of minimum weight.

Step 2: Enter cell.

Step 3: Reduce cell weight by 1.

Step 4: copy the modified weight of the cell.

Step 5: Perform transmissions.

Step 6: Go to step 0.

Fig. 4. Area into Hexa- Grids 


\section{Conclusion}

The proposed model enables the network to propagate the nodes into the disaster areas according to grid cells distribution. This is done with priority given to each cell as a leading value for the nodes to spread accordingly. This enables the Ad hoc nodes to repair any distortion or interruption in the communication channels in the disaster areas. In which should help in the recovery and rescuing in such areas.

For future directions, the model will be prepared using networks simulation, taking into account street maps representation. Moreover, the mobility model will be incorporated into different routing mechanisms to evaluate it role in improving performance levels. Finally, the model shall be used with media access control protocols to reach higher performance and fairness in channel sharing.

\section{$5 \quad$ References}

[1] Ray, N., Turuk, A. (2016). A framework for post-disaster communication using wireless ad hoc Networks. INTEGRATION the VLSI journal, retrieved on: https://doi.org/10.1016/j.vlsi.2016.11.011

[2] Portmann, M., Pirzada, A.A. (2008). Wireless mesh networks for public safety and crisis management applications, IEEE Internet Comput. 12 (1) 18-25. IEEE. https://doi.org/10.1109/MIC.2008.25

[3] Boukerche, A. Turgut, B., Aydin, N., Ahmad, M., Boloni, L., Turgut, D. (2011). Routing protocols in ad hoc networks: A survey, Comput. Netw. 55 (13) 3032-3080. https://doi.org/10.1016/j.comnet.2011.05.010

[4] M. Tarique, K.E. Tepe, S. Adibi, S. Erfani, Survey of multipath routing protocols for mobile ad hoc networks, J. Netw. Comput. Appl. 32 (6) (2009) 1125-1143. Elsevier https://doi.org/10.1016/j.jnca.2009.07.002

[5] Lee, H., Jeon, D. (2015). A Mobile Ad-Hoc network multipath routing protocol based on biological attractor selection for disaster recovery communication, The Korean Institute of Communications Information Sciences. ICT Express 1 (2015) 86-89. https://doi.org/10.1016/j.icte.2015.10.001

[6] Dressler, F. , and Akan, O.B., (2010). A survey on bio-inspired networking, Comput. Netw. 54 (6). https://doi.org/10.1016/j.comnet.2009.10.024

[7] Briante, O. , Loscri, V., Pace, P., Ruggeri, G., Zema, N. (2015). COMVIVOR: an evolutionary communication framework based on survivors' devices reuse, Wirel. Pers. Commun. 85 (4) 2021-2040. https://doi.org/10.1007/s11277-015-2888-y

[8] Schroedl , S., Wagstaff, K., Rogers, S., Langley, P., Wilson, C., (2004). Mining GPS traces for map refinement, Data Min. Knowl. Discov. 9 (1) 59-87. https://doi.org/10.1023/ B:DAMI.0000026904.74892.89

[9] Martín-Campillo, A., Crowcroft, J., Yoneki, E., Martí, R. (2013). Evaluating opportunistic networks in disaster scenarios, J. Netw. Comput. Appl. 36 (2) 870-880. https://doi.org/10.1016/j.jnca.2012.11.001

[10] Kikuchi, Y., Shibata, Y. (2015). Mobile cloud computing for distributed disaster information system in challenged communication environment, in: Advanced In- formation Networking and Applications Workshops (WAINA), 2015 IEEE 29th International Conference on, IEEE, pp. 512-517. https://doi.org/10.1109/WAINA.2015.82 
[11] Chenji, H., Zhang, W., Stoleru, R., Arnett, C. (2013). Distressnet: a disaster response system providing constant availability cloud-like services, Ad Hoc Netw. 11 (8) 2440-2460. https://doi.org/10.1016/j.adhoc.2013.06.008

\section{Authors}

Saher Manaseer has a PhD in Computer Science from the Department of Computing Science at the University of Glasgow. His main area of research is Computer Networks and Embedded Systems. Currently, Dr. Manaseer is an active researcher in the field of Mobile Ad Hoc Networks. More specifically, his research on MANETs is focused on developing MAC layer protocols. Before obtaining his $\mathrm{PhD}, \mathrm{He}$ got his Masters in Computing Science from the University of Jordan where his research in that era was focused on Software Engineering. Since he earned his undergraduate degree in Computer science, Dr. Manaseer has worked in two parallel directions, being Training and software development.

Afnan Alawneh is a member of Jordan University staff, works in the University of Jordan library, and as a researcher, quality assurance representative, got her B.Sc. in Information technology in 2005, and completed an MBA in 2014, both form the University of Jordan.

Article submitted 05 May 2017. Published as resubmitted by the authors 07 June 2017. 